ARTÍCULO DE INVESTIGACIÓN

\title{
Opciones reales para determinar el turno óptimo en sistemas silvopastoriles: análisis de inversión
}

\author{
Real options for determining optimum time \\ systems sylvopastoral: investment analysis
}

\author{
Gastón Milanesi, ${ }^{1}$ Diego Ricardo Broz ${ }^{2}$ \\ y Guillermo Bruno Woitschach ${ }^{3}$
}

\begin{abstract}
RESUMEN
El trabajo propone analizar y valorar, desde la perspectiva económica-financiera, el momento óptimo de cosecha en un sistema silvopastoril, utilizando las Opciones Reales (OR) como herramienta para la toma de decisiones estratégicas. Ésta se basa en un proceso estocástico para la proyección de precios, mientras que el Valor Actual Neto (VAN) utiliza una tasa fija en todo el proyecto. Los resultados mediante OR indican talar a los 18 años con un VOR (Valor Opción Real) \$AR 1088339 frente a la alternativa VAN de talar a los 11 años y \$AR 511914.
\end{abstract}

PALABRAS CLAVE

Flujo de fondos, momento óptimo, opciones reales, rejilla binomial.

\begin{abstract}
In this work we assess, from an economic and financial perspective, the optimal time of timber harvest in joint forestry-pasture systems. For this we apply a Real Options (RO) approach as a tool of strategic decision making. It assumes a stochastic process of price projections and is compared with the present value (NPV) computed on the basis of a fixed rate all along the project. The results obtained by means of the RO approach recommend cutting at 18 years with a ROV (Real Options Valuation) of \$AR 1088339 (ARG) against the alternative NPV assessment that recommends cutting at 11 years and yields \$AR 511914.
\end{abstract}

KEY WORDS

Cash flow, optimal timing, real options, binomial lattice. Argentina. C. e.: milanesi@uns.edu.ar Blanca, Argentina. C. e.: diego.broz@uns.edu.ar 


\section{INTRODUCCIÓN}

Los sistemas agroforestales o mixtos, específicamente los sistemas silvopastoriles (SSP) están teniendo auge en Argentina debido a la flexibilidad en el flujo económico, permitiendo la generación de ingresos anuales a partir del cuarto año del establecimiento; producto del componente ganadero y del ingreso final del componente forestal (Fassola et al., 2004). Son varios los autores que afirman bondades del SSP. Musálem (2002) menciona que los sistemas pueden estar en zonas fértiles, como también en zonas áridas, mejorando la productividad de estas últimas; Blasco et al. (2005) lo plantean como una estrategia económica para combatir el vinal (Prosopis ruscifolia) frente a métodos tradicionales; Cerruto et al. (2007) plantea que los SSP permiten abatir la erosión en comparación a una pastura degradada, aportando aumento de la viabilidad financiera; para Soares et al. (2003) los SSP son una alternativa de diversificación de rentas y recuperación ambiental. Se concluye que los SSP tienen importancia desde el punto de vista económico y ecológico, por ello se requieren herramientas para su gestión como una manera de tomar decisiones óptimas.

En este tipo de inversiones se asocia a la maduración del proyecto con la rotación, éste es un término relacionado con el tiempo que lleva una población en ser cortada (Schneider, 2002), convirtiendo el capital en renta. El mismo autor menciona que la duración de la rotación es definida por la meta técnica de la empresa, siendo los criterios tanto económicos como biológicos. Bettinger et al. (2009) enumeran siete tipos de rotación: física, técnica, silvicultural, biológica, por generación de ingresos, económica-financiera y valoración del crecimiento porcentual.

En general, el método empleado para valorar activos reales es el descuento de flujo de fondos (DFF) (Copeland et al.,
2000; Pratt y Grabowski, 2008). No obstante, este método no incorpora en el valor los múltiples cursos de acción o flexibilidad estratégica, propios de la vida proyectada e incierta de la inversión en cuestión (Mun, 2005). Valorar la flexibilidad estratégica de una inversión se logra adaptando la Teoría de Opciones para instrumentos financieros (Black y Scholes, 1973; Merton, 1973) a problemas de inversión en activos reales, dando lugar a los modelos de Valoración de Opciones Reales (VOR) (Dixit y Pindyck, 1994; Trigeorgis, 1997). En la literatura financiera el primer modelo corresponde a valorar la opción de crecimiento (Myers, 1977), a partir de este trabajo se han desarrollado diferentes propuestas analíticas para el tratamiento de categorías específicas de opciones. A continuación se enumeran algunos trabajos seminales para cada tipo: a) opción de diferimiento (Mc Donald y Siegel, 1981; Paddock et al., 1988); b) opción de crecimiento (Pindyck, 1988; Trigeorgis, 1988; Smit, 1997); c) opción de abandono (Myers y Majd, 1990); d) opciones de expandir-contraer o extensión de la vida útil (Trigeorgis y Mason, 1987); e) opción de cierre temporario o corte del proceso productivo (Brennan y Schwartz, 1985); f) opción de intercambio (Margrabe, 1978; Kulatilaka, 1988); g) opciones financieras de insolvencia (Mason y Merton, 1985). Si bien los clásicos modelos plantean ecuaciones en diferencia estocástica en tiempo continuo, para el análisis estratégico de activos reales se emplea el método binomial (Cox et al., 1979), utilizando rejillas en lugar de árboles, dada su capacidad de recombinar nodos intermedios (Smith, 2005; Milanesi y Vigier, 2010).

En el campo forestal los pioneros en la aplicación de componentes estocásticos en cosecha fueron Malliaris y Brock (1982), Miller y Voltaire (1983), entre otros. Thomson (1992), así como Yin y Newman (1997) estudiaron el turno óptimo 
de cosecha, asumiendo que los precios siguen un movimiento geométrico browniano, estos últimos autores mencionan que los enfoques tradicionales, como la Metodología de Faustmann (Grainger, 1968), se limitan a un mundo determinista.

Para Sant'Anna y Nogueira (2010), la VOR es una herramienta más realista de evaluación que el valor actual neto (VAN), ya que la tasa de descuento permanece inalterable en la vida del proyecto al realizar el análisis. El autor recalca que, en valoraciones de concesiones forestales, la VOR presenta valores significativamente mayores que VAN, esto coincide con Moreira et al. (2000), quien menciona que los resultados obtenidos por vOR fueron $140 \%$ superiores a los calculados por VAN y más realistas.

Soares et al. (2003) mencionan que la tasa de descuento afecta significativamente el VAN, siendo más rentable el proyecto cuanto menor sea la tasa. La tasa de descuento no se elabora mediante un procedimiento cuantitativo riguroso, sino mediante un análisis cualitativo integral, no riguroso o de poca consistencia, condicionado por costo de oportunidad, grado de riesgo del proyecto, costo del dinero, entre otros; afectando directamente en la toma de decisión sobre proyecto. Entre los trabajos que proponen alguna tasa encontramos a Monicault y Delvalle (2009) con una tasa de descuento de $12 \%$ obteniendo un VAN de \$AR 9357 y un turno de 12 años en el estudio de un SSP en la Provincia del Chaco, Argentina; Chidiak et al. (2003) plantea un estudio de factibilidad de inversiones forestales en la Patagonia con tasas de $8 \%$ a $12 \%$, además menciona que por debajo de $8 \%$ el VAN es negativo; Colcombet et al. (2010) concluye que un proyecto forestal a $152 \mathrm{~km}$ de la industria resiste holgadamente tasas de descuento de $8 \%$ anual en la Provincia de Misiones; Fassola et al.
(2004) mencionan Ingresos Anuales Equivalentes (IAE) mayores a \$AR 500 con una tasa de $5 \%$, siendo superior el beneficio de los SSP frente a forestación pura, invernada de novillos, maíz y soja.

En este trabajo se plantea la necesidad de aplicar el enfoque de la vor en el ejercicio de la tala en un sistema de producción mixto forestal-ganadero, comparando cada año, a partir del décimo (madurez tecnológica) hasta el vigésimo (máximo horizonte de planificación), el valor obtenido, producto de la cosecha en los nodos de la rejilla binomial (año $t$ ) versus el valor actual neto esperado de talar en el próximo año (año $t+1)$. La comparación periodo a periodo es relevante porque estos valores se caracterizan por ser cambiantes en función del tiempo y riesgo de las variables. El caso de análisis se corresponde con un escenario forestal simulado y un nivel de producción ganadera acorde con el patrimonio forestal, el cual permitirá determinar la viabilidad económica del proyecto de inversión para el noreste de la República Argentina, mediante la aplicación de la VOR y la comparación con el VAN.

\section{OBJETIVOS}

El primero objetivo de este trabajo fue la determinación de la madurez del proyecto de inversión para un sistema de producción mixto forestal-ganadero para el noreste argentino, el cual se corresponde con la determinación del turno óptimo de cosecha mediante la aplicación de OR. Como segundo objetivo se planteó la comparación de resultados entre un escenario evaluado mediante la VOR y por otro lado el VAN. Como tercer y último objetivo se planteó fundamentar el uso de la VOR como metodología de evaluación económica-financiera de un sistema de producción mixto frente al uso del VAN. 


\section{MATERIALES Y MÉTODOS}

\section{Valoración del componente forestal en el SSP}

Para evaluar el proyecto de inversión, se proyectaron a partir de los años 10 y hasta un horizonte de planificación de 20 años, los ingresos y egresos de cada una de las componentes del sistema para luego aplicar la VOR y el VAN y, consecuentemente, tomar las decisiones estratégicas que generaron mayor beneficio. La evolución de la masa forestal fue obtenida mediante el software Simulador Forestal (Crechi et al., 2005) y la producción ganadera (carga por hectárea) se supuso constante desde el inicio hasta el final del horizonte de planificación.

La volatilidad se estimó a partir de la serie histórica de precios correspondientes a los subproductos de la explotación forestal, considerados estos como cartera de productos y aplicando los conceptos de la Teoría Moderna de la Cartera (TMC) (Markowitz, 1991). ${ }^{4}$ Por otro lado, para el componente ganadero se utilizó solamente la serie histórica, ya que a diferencia del forestal, existe un sólo producto comercial. Para calcular el precio promedio y la variabilidad de la mezcla de subproductos obtenidos, aplicando los conceptos de la TMC, estimando el precio medio se utilizó la ecuación (1), el riesgo del precio es determinado por la ecuación (2).

$$
P_{0}=\sum_{i}^{n} x_{i} P_{i}
$$

4 La Teoría de la Cartera (Modern Portfolio Theory) es aplicable a todo tipo de carteras de activos. En este caso, el precio promedio de la masa forestal es el promedio ponderado del promedio observado de cada subproducto. La volatilidad es representada por la suma ponderada de los desvíos observados ajustados por la covarianza (correlación) precio correspondiente a la familia de subproductos. donde:

$P_{i}$ : precio promedio de cada subproducto

$x_{i}$ : participación en el total producido

$P_{o}$ : precio promedio

$\sigma_{c}=\sum_{i=1 ; j=1}^{n} x_{i}^{2} \sigma_{i}^{2}+x_{j}^{2} \sigma_{j}^{2}+2\left(x_{i} x_{j}\right) \rho_{i j} \sigma_{i} \sigma_{j}$

donde:

$\sigma_{c}$ : riesgo del precio de la cartera de productos

$x_{i}$ y $x_{j}$ : participaciones de cada subproducto

$\sigma_{i}^{2}$ y $\sigma_{j}^{2}$ : varianzas de los precios de los subproductos

$\sigma_{i}$ y $\sigma_{j}$ : desvíos de los precios

$\rho_{i j} \quad$ : coeficiente de correlación de precios

Usando el precio promedio de la ecuación (2), se calcularon los flujos de fondos libres con la ecuación (3).

$$
F F_{t}=\left[P_{0} \times \operatorname{vol}_{t}\right] \times\left(1-C_{t}\right) \times(1-I)
$$

donde:

$F F_{t}$ : flujos de fondos libres

vol $l_{t}$ :producción esperada en cada periodo del horizonte de planificación obtenido mediante simulación

$C_{t}$ : porcentaje que representan los costos de producción (variables y de estructura) sobre los ingresos por ventas, calculado mediante la ecuación (4).

$I$ : tasa marginal de impuesto a las ganancias.

La tasa marginal de impuesto a las ganancias $I$ fue de $35 \%$.

$$
C_{t}=\frac{c_{t}}{\left[P_{0} \times \text { vol }_{t}\right]}
$$




$$
V A N_{f t}=F F_{t} \times e^{-k t}
$$

El valor intrínseco de la explotación sin flexibilidad estratégica se estimó con la ecuación (5), siendo su expresión el valor actual de los flujos de fondos derivados de la tala en cada uno de los años, descontados a la tasa ajustada por riesgo sobre inversiones equivalentes. ${ }^{5}$ La estructura de la tasa para mercado emergente, poco diversificado y segmentado (Damodaran, 2002), surgió de ajustar el rendimiento de mercado por la volatilidad total, en este caso dada por el desvío del precio. La ecuación (6) muestra la formulación de la tasa ajustada por riesgo.

$$
k=(1+r m) \times\left(1+\sigma_{c} \sqrt{\Delta t}\right)
$$

donde:

rm : adicional por riesgo de mercado

$\sigma_{c}$ : variación del precio

$\Delta t$ : relación entre $T / n$, siendo esto la longitud de cada paso del modelo

$k \quad$ : relación porcentual

La proyección del precio de venta promedio de los subproductos forestales sigue un proceso estocástico del tipo Geométrico Browniano. Se trabajó en tiempo discreto y, para la elaboración de una rejilla binomial, ${ }^{6}$ se utilizaron los parámetros del

5 La tasa por riesgo equivalente se calcula aplicando los clásicos modelos como el CAPM (Capital Asset Pricing Model), APM (Arbitrage Pricing Model); MPM (Multifactor Pricing Model) y sus adecuaciones a mercados emergentes.

$6 \quad$ Las rejillas binomiales son empleadas debido a su capacidad de recombinar nodos e incorporar las propiedades propias del triángulo de Pascal. Los árboles binomiales explicitan gráficamente el problema de manera más eficiente. No obstante, no permiten recombinar, lo que torna su cálculo computacionalmente más complejo que las rejillas. Si $n$ representa los periodos o intervalos de tiempo, la cantidades de nodos finales de una rejilla es de $n+1$ y de nodos intermedios $n(n+1) / 2$. En los árboles binomiales, en cambio, estos valores son $2 n-1$ y $2 n-1$, respectivamente (Smith, 2005). modelo CRR (Cox et al., 1979) mediante las ecuaciones (7) y (8).

$$
\begin{gathered}
u=e^{\left(\sigma_{c} \sqrt{t}\right)} \\
d=e^{-\left(\sigma_{c} \sqrt{t}\right)}
\end{gathered}
$$

Los movimientos de ascenso $u$ y descenso $d$ se calcularon utilizando el desvío de la cartera de precios calculado con la ecuación (2). El precio de cada nodo padre $P_{i j}$ de la rejilla en $t-1$ genera los precios ascendentes $i$ y descendentes $j$ en $t$ por medio de la ecuación (9).

$$
P_{i j(t)}=\left\lfloor P_{i(t-1)} \times u ; P_{j(t-1)} \times d\right\rfloor
$$

Una vez proyectado el proceso estocástico del precio por medio de las ecuaciones (7) y (8), se calculó nuevamente el flujo de fondos para cada periodo $t$ y cada nodo $(i, j)$ mediante la ecuación (10).

$$
F F_{i j(t)}=\left\lfloor P_{i j(t)} \times \operatorname{vol}_{t}\right\rfloor \times\left(1-C_{t}\right)
$$

Con los flujos de fondos para cada nodo de la rejilla se construyeron las OR, para ello fue necesario definir el precio de ejercicio de la opción. La decisión a que se enfrenta el agente es seleccionar el momento de madurez de la inversión, que se corresponde con la determinación del turno óptimo de cosecha del SSP, para lo cual se debe seguir un criterio de maximización de beneficios, conjugando el factor tiempo y el riesgo que emerge de las variables precio de los subproductos. Los flujos de fondos proyectados en cada

$7 \quad$ Los nodos pueden interpretarse como posibles escenarios futuros; a mayor intervalo de tiempo mayor cantidad de nodos debido a la mayor incertidumbre. Así, para el periodo $t=1$ la cantidad de nodos de la rejilla es de 2 (dos escenarios) mientras que para $t=10$ la cantidad es 11 . 
escenario de la rejilla fueron comparados con el valor esperado del próximo año, según la expresión (11).

$$
X_{t+1}=\sum_{i=1 ; j=1}^{n} F F_{i j(t+1)} \times q_{i j(t+1)}
$$

Donde $X_{t+1}$ es el promedio de los flujos de fondos en cada nodo del próximo periodo $F F_{i j(t+1)}$ ponderado por la probabilidad asociada $q_{i j(t+1)}$, siendo las probabilidades asociadas a cada nodo dadas por la ecuación (12).

$$
q_{i j(t+1)}=\frac{n !}{m !(n-m !)} \times\left[p^{m} \times\left(1-p^{n-m}\right)\right]
$$

En este caso, los éxitos $m$ estuvieron dados por los movimientos de ascenso (subas) del precio sobre el total de observaciones $n$ y condicionados por los coeficientes equivalente-ciertos $p$, estimados con la ecuación (13).

$$
p=\frac{(1+r)^{\Delta t}-d}{u-d}
$$

Donde $r$ representa la tasa libre de riesgo y los movimientos $(u ; d)$, surgen de las ecuaciones (7) y (8) y $\Delta t$ se definió en la ecuación (6). El valor esperado $V_{i j(t)}$ surgió de comparar en cada periodo, el valor correspondiente a cada nodo $F F_{i j(t)}$ con el valor esperado en el próximo año; $X_{t+1} \times e^{-r \Delta t}$ matemáticamente el problema se resume en la expresión (14).

$$
V_{i j(t)}=\left\{\begin{array}{l}
F F_{i j(t)}, \text { si } F F_{i j(t)}>X_{t+1} \times e^{-r \Delta t} \\
0, \quad \text { sino }
\end{array}\right.
$$

El valor de la opción en el momento inicial $(t=0)$ requiere de utilizar el procedimiento recursivo sobre la rejilla (Milanesi y
Viger, 2010; Milanesi, 2011) dado en la ecuación (15).

$$
V_{0 t}=\sum_{i=1 ; j=1 ; t}^{n}\left\{V_{i j(t)} \times q_{i j(t)}\right\} \times e^{-r \Delta t}
$$

Siendo $V_{0 t}$ el valor actual de la opción de talar en el momento $t$. El ejercicio de la opción en cada nodo (escenario) en $t$ se expresa por $V_{i j(t)}$. La probabilidad de alcanzar el nodo asociado es $q_{i j(t)}$. El factor de actualización desde el momento ( $t$ ) hasta el inicio $(t=0)$ es $e^{-r \Delta t}$.

\section{Valoración del componente ganadero en el SSP}

El análisis del componente ganadero fue análogo al del componente forestal. La particularidad de este componente es la generación de flujos de cajas anuales, donde hubo egresos por compra de terneros y costos de producción y un ingreso a los doce meses producto de la venta. El VAN se determinó con la ecuación (16)

$$
V A N_{g t}=\left(I G_{t}-E G_{t}\right) \times e^{-k G \times t}
$$

donde

$I G_{t}$ : ingreso por venta del componente ganadero al final del período $t$

$E G_{t}$ : egresos debido a costos operativos y compra del ganado al inicio de $t$

$k G$ : tasa ajustada por riesgo obtenida para el componente ganadero mediante la ecuación (17).

$$
k G=(1+r m) \times\left(1+\sigma_{g} \sqrt{\Delta t}\right)
$$

El precio inicial surgió de aplicar la ecuación (18).

$$
\bar{x}=\frac{\sum_{i=1}^{n} x_{i}}{n}
$$


donde:

$x_{i}$ : observación de precio mensual

$\bar{x}$ : media de la serie de precios

$n$ : observaciones.

El riesgo del precio $\sigma_{\mathrm{g}}$ se obtuvo a partir de la serie de precios mensuales aplicando la ecuación (19)

$$
\sigma_{g}=\sqrt[12]{\frac{1}{N} \sum_{n=1}^{N}\left[\left(x_{i+1}-x_{i}\right) / x_{i}-\bar{x}\right]}
$$

donde:

$N$ : número de observaciones

$\left(x_{i+1}-x_{i}\right) / x_{i}$ : variación relativa del precio

Para el cálculo de las tasas de ascenso y descenso $(u, d)$ se aplicaron las ecuaciones (7) y (8). Se realizaron las proyecciones de los precios estocásticos mediante la ecuación (9). Se calculó el nuevo flujo de fondo mediante la ecuación (20).

$$
F F_{i j(t)}=\left(P_{i j(t)} \times Q_{t}\right)-E G_{t}
$$

En este caso:

$P_{i j(t)}$ : precio promedio

$Q_{t}$ : cantidad total (en $\overline{\mathrm{kg}}$ ) de venta de ganado

$E G_{t}$ : egreso

Posteriormente, se calculó la probabilidad para cada nodo mediante la ecuación (12). Se obtuvo el flujo de fondo en función al equivalente cierto (21) que permitiría obtener $V_{i j(t)}$ mediante la ecuación (14).

$$
X_{t}=\sum_{1}^{n} F F_{i j(t)} \times q_{i j(t)}
$$

El valor de la opción $V_{0 t}$ se obtuvo aplicando la ecuación (15).

\section{Análisis de sistema mediante la vor y el VAN}

En esta etapa convergen los resultados con el objeto de un análisis integral del sistema. Para la obtención de un $V A N$ total $\left(V A N_{T}\right)$ se sumó en $t$ los $V A N_{f} \mathrm{y}_{V A N_{g}}$, de la misma manera se procede con el ${ }^{g} V O R$ total $\left(V O R_{T}\right)$ ya que los valores son aditivos (Rocabert, 2007). En las ecuaciones (22) y (23) se puede ver la expresión matemática.

$$
\begin{gathered}
V A N_{T t}=V A N_{f t}+V A N_{g t} \\
V O R_{T t}=V O R_{f t}+V O R_{g t}
\end{gathered}
$$

El turno óptimo (TO) de cosecha forestal en SSP se da cuando $V_{T O R_{T(t)}}>V O R_{T(t+1)}$, su expresión matemática es la (24); mientras que en el caso del $V A N$, la cosecha se da cuando se obtiene el máximo $V A N_{T t}$.

$$
\operatorname{VOR}_{T}=\left\{\begin{array}{lll}
\operatorname{VOR}_{T(t)}, & \text { si } & \operatorname{VOR}_{T(t)}>\operatorname{VOR}_{T(t+1)} \rightarrow \text { Talar } \\
0, & \text { si } & \text { no } \rightarrow \text { No Talar }
\end{array}\right.
$$

Si el valor de los flujos de fondos libres $F F_{i j(t)}$ supera al valor actual esperado del próximo año $X_{t+1} \times e^{-r \Delta t}$; se ejerce la tala, siendo su valor de $V_{i j}=F F_{i j(t)}$. Caso contrario, si el va-lor esperado para el próximo año supera los flujos de fondos libres del nodo, el resultado es de $V_{i j}=0$ difiriendo la tala al año próximo (Plantinga, 1998). La decisión en cuestión se asimila a una opción exótica barrera o barrier option (Hull, 2005).

\section{Valoración de un sistema silvopastoril: caso de aplicación}

Se propuso analizar el turno óptimo de cosecha de un proyecto de inversión mixto mediante la aplicación de opciones reales a un predio de 39,8 ha sobre el cual se reforestaría con Pinus taeda L. La producción 
Tabla 1. Egresos e ingresos del componente ganadero para un ciclo de 12 meses.

\begin{tabular}{lr}
\hline Compra & 94.656 \\
\hline MO & 91000 \\
Alimento y sanidad & 20776 \\
Costo Total & 206432 \\
Ingreso por venta & 336240 \\
\hline
\end{tabular}

Fuente: Elaboración propia. Sistema Integrado de Información Agropecuaria y Subsecretaría de Agricultura. Moneda: \$AR

se estimó mediante la simulación del crecimiento de la masa boscosa. La franja de edad evaluada fue a partir del año $10(t=0)$, tomando un horizonte de planificación de 10 años. Los objetivos del cultivo y manejo del rodal estaban alineados con aquellos tendientes a obtener subproductos de alto valor; para ello, los destinos eran la industria de laminado y la industria de aserrío de grandes dimensiones $(\varnothing>30 \mathrm{~cm})$; el resto de los subproductos eran para aserrío de menores dimensiones $(\varnothing<30 \mathrm{~cm})$ y pulpa celulósica.

En la simulación del manejo de la masa forestal se previó el inicio del ciclo con 1600 plantas por hectárea, 3 intervenciones de aclareos (raleos) con una intensidad de $50 \%$ a $55 \%$ y periodicidad de 2 años entre intervención, lo que permite obtener madera de alto valor favoreciendo el volumen individual (Crechi et al., 2005). Se planificó la ejecución de podas, que serían realizadas de acuerdo con la obtención de un cilindro defectuoso menor a 12 $\mathrm{cm}$ y una altura total de poda de $6,5 \mathrm{~m}$.

La simulación se llevó a cabo mediante el software Simulador Forestal (Crechi et al., 2005). Se consideró un índice de sitio de $22 \mathrm{~m}$ (edad índice 15) y una densidad final de 190 árboles por hectárea. Los parámetros iniciales (año 10) del bosque fueron: altura media $16,4 \mathrm{~m}$; diámetro medio $29,9 \mathrm{~cm}$; área basal 13,5 $\mathrm{m}^{2}$ /ha, un volumen de $108,7 \mathrm{~m}^{3} /$ ha $y$ un IDR (Prodan et al., 1997) de 256.
El componente ganadero estuvo constituido por la raza Braford. Se estimó alrededor de 2 animales por hectárea requiriendo un total de 80 individuos con un peso inicial de aproximadamente 120 $\mathrm{kg}$ y un precio de compra de $\$ A R 9,86 / \mathrm{kg}$, el peso final para comercialización se estipula en $450 \mathrm{~kg}$ y un precio de venta de \$AR 9,34/kg. El manejo correspondió al tipo invernada con un ciclo de 12 meses. Los costos e ingresos se detallan en la tabla 1.

\section{RESULTADOS}

\section{Estimación de los flujos de fondos}

El precio promedio inicial surgió de aplicar la ecuación (1), arrojando un valor de \$AR 189 por tonelada y aplicando la ecuación (17) un valor de \$AR 6,16/kg de peso vivo para el periodo $t=0$. Para el riesgo de precio forestal se aplicó la ecuación (2), utilizando cuatro series históricas de precios correspondientes a cuatro clases diamétricas; ${ }^{8}$ para el riesgo de precio ganadero se aplicó la ecuación (19), utilizando una serie de precios históricos de ganado en pie $^{9}$, los valores obtenidos para

8 Fuente: Colegio de Ingenieros Forestales de Misiones (COIFORM). Datos mensuales para el Norte de la Provincia de Misiones entre los períodos julio de 2002 a diciembre de 2011.

9 Fuente: Sistema Integrado de Información Agropecuaria y Subsecretaría de Agricultura. Datos 
Tabla 2. Valor Actual Neto del sistema entre el año 10 y 20 , en gris se señala el turno.

\begin{tabular}{|c|c|c|c|c|c|c|c|c|c|c|c|}
\hline Año & 10 & 18 & 12 & 13 & 14 & 15 & 16 & 17 & 18 & 19 & 20 \\
\hline VAN Forestal & 378224 & 382105 & 360674 & 324062 & 283374 & 241650 & 202137 & 167130 & 136462 & 110305 & 88657 \\
\hline VAN Ganedaro & 129808 & 129808 & 129808 & 129808 & 129808 & 129808 & 129808 & $1298 C 8$ & 129808 & 129908 & 129808 \\
\hline VAN Iotal & 508032 & 611014 & 480482 & 453870 & 413182 & $371 \angle 58$ & 331046 & 296047 & 266270 & 240203 & 218.466 \\
\hline
\end{tabular}

Fuente: Elaboración propia. Moneda: \$AR

la cartera de subproductos forestales $\left(\sigma_{\mathrm{s}}\right)$ fueron de $13,57 \%$ y $38,3 \%\left(\sigma_{\mathrm{g}}\right)$ para el componente ganadero.

\section{Descuento de flujos de fondos}

Empleando los flujos de fondos se determinó el valor actual de las potenciales talas (ecuación (5)). La tasa ajustada por riesgo (ecuación (6)) fue de $28,39 \%{ }^{10}$ para el componente forestal y $34,58 \%{ }^{11}$ para el componente ganadero. Debido al crecimiento de la masa forestal, los mayores flujos de fondos se obtuvieron en el último periodo. Por la sensibilidad de los beneficios al factor de actualización, el criterio del descuento de flujos de fondos indicó que el mayor valor actual se produjo en el instante $t=1$ (año 11), el resultado sugiere que la tala se realice de manera inminente con un VAN de \$AR 511 914. En la tabla 2 se pude ver la evolución del VAN en el horizonte analizado. Se aprecia que el turno de cosecha del componente forestal en forma independiente es igual al turno analizado como sistema.

\section{Opciones reales}

Para la construcción del proceso estocástico de la variable de riesgo a los flujos de

mensuales para la Provincia de Misiones entre los períodos julio de 2008 a diciembre de 2011

10 Para ello se supuso un rendimiento de mercado de $25 \%$ (suponiendo nivel esperado de inflación).

11 Para ello se supuso un rendimiento de mercado de $25 \%$ (suponiendo nivel esperado de inflación). fondos se incorporó el riesgo de precio. Conforme fue expresado, este ascendió a $13,57 \%$ y $38,30 \%$ para el componente forestal y ganadero, respectivamente. El movimiento de ascenso y descenso, calculado mediante las ecuaciones (7) y (8), fue de $u=1,1453$ y $d=0,8731$ para el componente forestal y $u=1,4667 \mathrm{y}$ $d=0,6818$ para el componente ganadero. Para la construcción de la rejilla se aplicó la ecuación (10) y se estimaron los flujos de fondos mediante la ecuación (11). Mediante la ecuación (13), se determinaron las probabilidades binomiales para cada nodo siendo el precio de ejercicio determinado con la ecuación (12).

Los coeficientes equivalentes ciertos (ecuación (13)) para el componente forestal fueron $p=0,6545$ y $1-p=0,3455$ y para el componente ganadero $p=0,4707 \mathrm{y}$ $1-p=0,5293$. Conforme fue expuesto, se acepta la tala (o madurez de la inversión) en el nodo proyectado si los flujos de fondos (ecuación (10)) superan al valor actual esperado en el próximo ejercicio (ecuación (11)). Para estimar el valor actual, el precio de ejercicio se actualizó al tipo sin riesgo, Milanesi et al. (2012) sugiere una tasa $r=5 \%$ para la Argentina. Para este cálculo se aplica la ecuación (14).

Para estimar el periodo conveniente para la tala desde el punto de vista económico-financiero, la información de la rejilla se resumió en una medida de valor esperado (ecuación (15)). Los diferentes resultados obtenidos en cada nodo, deben ponderarse por sus probabilidades de ocurrencia (ecuación (12)), obteniendo de esta manera el valor del ejercicio (tala). Para llevarlos al mismo horizonte tempo- 


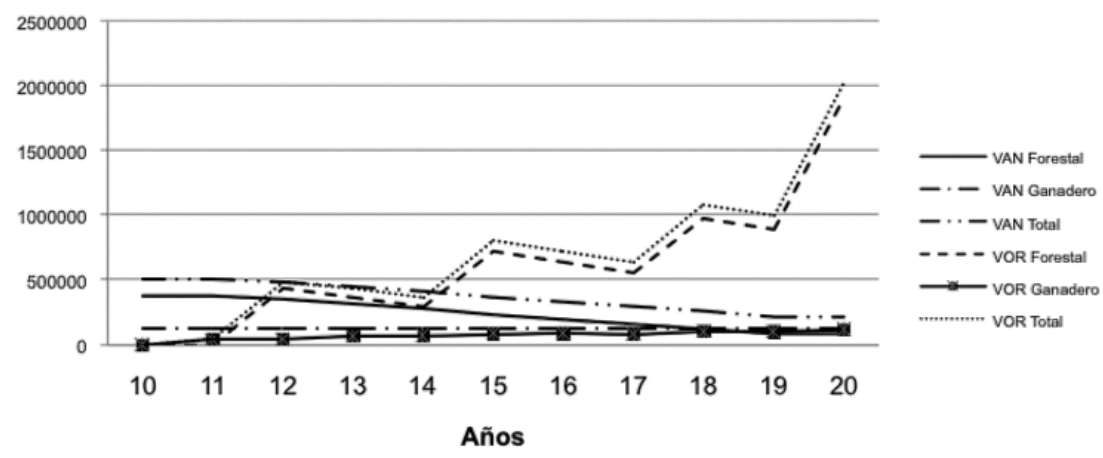

Figura 1. Comparación de la evolución del VAN y vOR por subsistema y total en \$AR

ral, se actualizó por el factor libre de riesgo $(r$ ). De la tabla 3 a la tabla 12 , se pueden ver los principales pasos para el desarrollo de la metodología para cada componente del sistema, mientras que en la tabla 13 se integran los resultados de las componentes del sistema para obtener un análisis integral. La decisión en juego en el último periodo se balanceó entre talar o abandonar, puesto que no había camino estratégico alternativo (venta o diferimiento).
A diferencia del criterio tradicional, el enfoque de opciones reales incorpora variables estocásticas (riesgo de precio) y la flexibilidad estratégica del ejercicio de talar. Entre los periodos $t=0$ hasta $t=9$, el máximo valor de ejercicio de la tala surgió en el periodo $t=8$ (año 18) con una VOR de \$AR 1088339.

Estos resultados difieren del criterio tradicional de descuento de flujo de fondos en más de $100 \%$.

Tabla 3. Proyección de precios promedios del componente forestal.

\begin{tabular}{|c|c|c|c|c|c|c|c|c|c|c|}
\hline 0 & 1 & 2 & 3 & 4 & 5 & 6 & 7 & 8 & 9 & 10 \\
\hline \multirow[t]{10}{*}{189} & 216,6 & 248,1 & 284,2 & 325,5 & 372,8 & 426,9 & 489,0 & 560,0 & 641,4 & 734,6 \\
\hline & 165,2 & 189,2 & 216,6 & 248,1 & 284,2 & 325,5 & 372,8 & 426,9 & 489,0 & 560,0 \\
\hline & & 144,2 & 165,2 & 189,2 & 216,6 & 248,1 & 284,2 & 325,5 & 372,8 & 426,9 \\
\hline & & & 125,9 & 144,2 & 165,2 & 189,2 & 216,6 & 248,1 & 284,2 & 325,5 \\
\hline & & & & 109,9 & 125,9 & 144,2 & 165,2 & 189,2 & 216,6 & 248,1 \\
\hline & & & & & 96,0 & 109,9 & 125,9 & 144,2 & 165,2 & 189,2 \\
\hline & & & & & & 83,8 & 96,0 & 109,9 & 125,9 & 144,2 \\
\hline & & & & & & & 73,2 & 83,8 & 96,0 & 109,9 \\
\hline & & & & & & & & 64 & 73,2 & 83,8 \\
\hline & & & & & & & & & 55,8 & 63,9 \\
\hline \multicolumn{3}{|c|}{ Precio Promedio: ecuaciones (1) y (9). } & & & & & & & & 48.7 \\
\hline
\end{tabular}


Tabla 4. Flujo de fondos libres del componente forestal.

\begin{tabular}{|c|c|c|c|c|c|c|c|c|c|c|}
\hline 0 & 1 & 2 & 3 & 4 & 5 & 6 & 7 & 8 & 9 & 10 \\
\hline \multirow[t]{10}{*}{581883} & 837539 & 1153997 & 1538143 & 2005086 & 2566176 & 3234572 & 4033541 & 4979071 & 6097691 & 7416481 \\
\hline & 638486 & 879733 & 1172581 & 1528549 & 1956287 & 2465829 & 3074912 & 3795723 & 4648486 & 5653847 \\
\hline & & 670652 & 893900 & 1165267 & 1491347 & 1879789 & 2344114 & 2893614 & 3543706 & 4310129 \\
\hline & & & 681452 & 888324 & 1136907 & 1433030 & 1787002 & 2205905 & 2701493 & 3285764 \\
\hline & & & & 677201 & 866705 & 1092450 & 1362295 & 1681640 & 2059444 & 2504855 \\
\hline & & & & & 660720 & 832813 & 1038526 & 1281974 & 1569987 & 1909540 \\
\hline & & & & & & 634883 & 791705 & 977294 & 1196857 & 1455710 \\
\hline & & & & & & & 603545 & 745026 & 912407 & 1109739 \\
\hline & & & & & & & & 567960 & 695560 & 845994 \\
\hline & & & & & & & & & 530250 & 644931 \\
\hline \multicolumn{3}{|c|}{ Flujo de Fondo: ecuación (10) } & & & & & & & & 491654 \\
\hline
\end{tabular}

Fuente: Elaboración propia. Moneda: \$AR

Tabla 5. Probabilidades asociadas a cada nodo

\begin{tabular}{|c|c|c|c|c|c|c|c|c|c|c|}
\hline 0 & 1 & 2 & 3 & 4 & 5 & 6 & 7 & 8 & 9 & 10 \\
\hline \multirow[t]{11}{*}{$100,00 \%$} & $65,45 \%$ & $42,84 \%$ & $28,04 \%$ & $18,35 \%$ & $12,01 \%$ & $7,86 \%$ & $5,14 \%$ & $3,37 \%$ & $2,20 \%$ & $1,44 \%$ \\
\hline & $34,55 \%$ & $45,23 \%$ & $44,40 \%$ & $38,75 \%$ & $31,70 \%$ & $24,90 \%$ & $19,01 \%$ & $14,22 \%$ & $10,47 \%$ & $7,61 \%$ \\
\hline & $100,00 \%$ & $11,94 \%$ & $23,44 \%$ & $30,68 \%$ & $33,47 \%$ & $32,86 \%$ & $30,11 \%$ & $26,27 \%$ & $22,11 \%$ & $18,09 \%$ \\
\hline & & $100,00 \%$ & $4,12 \%$ & $10,80 \%$ & $17,67 \%$ & $23,13 \%$ & $26,49 \%$ & $27,74 \%$ & $27,23 \%$ & $25,46 \%$ \\
\hline & & & $100,00 \%$ & $1,43 \%$ & $4,66 \%$ & $9,16 \%$ & $13,98 \%$ & $18,30 \%$ & $21,56 \%$ & $23,52 \%$ \\
\hline & & & & $100,00 \%$ & $0,49 \%$ & $1,93 \%$ & $4,43 \%$ & $7,73 \%$ & $11,38 \%$ & $14,90 \%$ \\
\hline & & & & & $100,00 \%$ & $0,17 \%$ & $0,78 \%$ & $2,04 \%$ & $4,01 \%$ & $6,56 \%$ \\
\hline & & & & & & $100,00 \%$ & $0.06 \%$ & $0,31 \%$ & $0,91 \%$ & $1,98 \%$ \\
\hline & & & & & & & $100,00 \%$ & $0,02 \%$ & $0,12 \%$ & $0,39 \%$ \\
\hline & & & & & & & & $100,00 \%$ & $0,01 \%$ & $0,05 \%$ \\
\hline & & & & & & & & & $100,00 \%$ & $0,00 \%$ \\
\hline \multicolumn{3}{|c|}{ Probabilidades: ecuación (13) } & & & & & & & & $100,00 \%$ \\
\hline
\end{tabular}

Fuente: Elaboración propia

Tabla 6. Comparación del valor correspondiente a cada nodo $\left(F F_{i j(t)}\right)$ con el valor esperado en el próximo año $\left(X_{t+1} \times e^{-r \Delta t}\right)$

\begin{tabular}{|c|c|c|c|c|c|c|c|c|c|c|}
\hline 0 & 1 & 2 & 3 & 4 & 5 & 6 & 7 & 8 & 9 & 10 \\
\hline 581883 & 768763 & 972255 & 1189488 & 1423260 & 1671958 & 1934388 & 2214120 & 2508712 & 2820042 & 3148299 \\
\hline 0 & 1 & 2 & 3 & 4 & 5 & 6 & 7 & 8 & 9 & 10 \\
\hline \multirow[t]{10}{*}{ - } & - & 1153997 & 1538143 & 2005086 & 2566176 & 3234572 & 4033541 & 4979071 & 6097691 & 7416481 \\
\hline & & - & $\cdot$ & $\cdot$ & 1956287 & 2465829 & 3074912 & 3795723 & 4648486 & 5653847 \\
\hline & & & - & $\cdot$ & $\cdot$ & $\cdot$ & - & 2893614 & 3543706 & 4310129 \\
\hline & & & - & - & - & - & - & - & - & 3285764 \\
\hline & & & & - & - & - & - & - & - & 2504855 \\
\hline & & & & & - & - & - & - & . & 1909540 \\
\hline & & & & & & - & - & - & $\cdot$ & 1455710 \\
\hline & & & & & & & - & - & - & 1109739 \\
\hline & & & & & & & & - & - & 845994 \\
\hline & & & & & & & & & - & 644931 \\
\hline Comparacic & ada period & tación (14) & & & & & & & & \\
\hline
\end{tabular}

Fuente: Elaboración propia. Moneda: \$AR 
Tabla 7. Valor de la Opción Real para el componente forestal

\begin{tabular}{|c|c|c|c|c|c|c|c|c|c|c|}
\hline 0 & 1 & 2 & 3 & 4 & 5 & 6 & 7 & 8 & 9 & 10 \\
\hline \multirow[t]{11}{*}{ - } & - & 494319 & 431222 & 367908 & 308173 & 254229 & 207490 & 167633 & 134363 & 106958 \\
\hline & - & - & - & - & 620115 & 613883 & 584525 & 539708 & 486664 & 430448 \\
\hline & & - & - & - & - & - & - & 760212 & 783426 & 779546 \\
\hline & & & - & . & - & - & - & - & - & 836601 \\
\hline & & & & - & - & . & - & - & - & 589203 \\
\hline & & & & & - & - & - & - & - & 284547 \\
\hline & & & & & & - & - & - & - & 95429 \\
\hline & & & & & & & . & - & - & 21946 \\
\hline & & & & & & & & - & - & 3312 \\
\hline & & & & & & & & & - & 296 \\
\hline & & & & & & & & & & - \\
\hline - & . & 447279 & 371156 & 301218 & 722951 & 643113 & 558124 & 983730 & 895519 & 1909532 \\
\hline
\end{tabular}

Fuente: Elaboración propia Moneda: \$AR

Tabla 8. Proyección de precios promedios del componente ganadero

\begin{tabular}{|c|c|c|c|c|c|c|c|c|c|c|}
\hline 0 & 1 & 2 & 3 & 4 & 5 & 6 & 7 & 8 & 9 & 10 \\
\hline \multirow[t]{10}{*}{6,16} & 9,04 & 13,26 & 19,45 & 28,52 & 41,83 & 61,35 & 89,98 & 131,98 & 193,57 & 283,90 \\
\hline & 4,20 & 6,16 & 9,04 & 13,26 & 19.45 & 28,52 & 41,83 & 61,35 & 89.98 & 131,98 \\
\hline & & 2,87 & 4.20 & 6,16 & 9.04 & 13,26 & 19,45 & 28,52 & 41,83 & 61,35 \\
\hline & & & 1,95 & 2,87 & 4,20 & 6,16 & 9,04 & 13,26 & 19,45 & 28,52 \\
\hline & & & & 1,33 & 1,95 & 2,87 & 4,20 & 6,16 & 9,04 & 13.26 \\
\hline & & & & & 0.91 & 1,33 & 1,95 & 2,87 & 4,20 & 6,16 \\
\hline & & & & & & 0,62 & 0,91 & 1,33 & 1,95 & 2,87 \\
\hline & & & & & & & 0,42 & 0,62 & 0.91 & 1,33 \\
\hline & & & & & & & & 0.29 & 0.42 & 0.62 \\
\hline & & & & & & & & & 0,20 & 0,29 \\
\hline Precic & ecuac & $y(19)$ & & & & & & & & 0,13 \\
\hline
\end{tabular}

Fuente: Elaboración propia. Moneda: \$AR

Tabla 9. Flujo de fondos libres del componente ganadero

\begin{tabular}{|c|c|c|c|c|c|c|c|c|c|c|}
\hline 0 & 1 & 2 & 3 & 4 & 5 & 6 & 7 & 8 & 9 & 10 \\
\hline \multirow[t]{10}{*}{15448} & 118995 & 270864 & 493608 & 820301 & 1299454 & 2002218 & 3032946 & 4544693 & 6761939 & 10013924 \\
\hline & -55151 & 15448 & 118995 & 270864 & 493608 & 820301 & 1299454 & 2002218 & 3032946 & 4544693 \\
\hline & & -103287 & -55151 & 15448 & 118995 & 270864 & 493608 & 820301 & 1299454 & 2002218 \\
\hline & & & -136106 & -103287 & -55151 & 15448 & 118995 & 270864 & 493608 & 820301 \\
\hline & & & & -158483 & -136106 & -103287 & -55151 & 15448 & 118995 & 270864 \\
\hline & & & & & -173740 & -158483 & -136106 & -103287 & -55151 & 15448 \\
\hline & & & & & & -184142 & -173740 & -158483 & -136106 & -103287 \\
\hline & & & & & & & -191234 & -184142 & -173740 & -158483 \\
\hline & & & & & & & & -196070 & -191234 & -184142 \\
\hline & & & & & & & & & -199367 & -196070 \\
\hline \multicolumn{3}{|c|}{ Flujo de Fondo: ecuación (20) } & & & & & & & & -201615 \\
\hline
\end{tabular}

Fuente: Elaboración propia. Moneda: \$AR 
Tabla 10. Probabilidades asociadas a cada nodo del componente ganadero

\begin{tabular}{|c|c|c|c|c|c|c|c|c|c|c|}
\hline 0 & 1 & 2 & 3 & 4 & 5 & 6 & 7 & 8 & 9 & $1000,00 \%$ \\
\hline \multirow{11}{*}{$100,00 \%$} & $47,07 \%$ & $22,16 \%$ & $10,43 \%$ & $4,91 \%$ & $2,31 \%$ & $1,09 \%$ & $0,51 \%$ & $0.24 \%$ & $0,11 \%$ & $0,05 \%$ \\
\hline & $52,93 \%$ & $49,83 \%$ & $35,18 \%$ & $22,08 \%$ & $12,99 \%$ & $7,34 \%$ & $4,03 \%$ & $2,17 \%$ & $1,15 \%$ & $0,60 \%$ \\
\hline & $100,00 \%$ & $28,01 \%$ & $39,56 \%$ & $37,24 \%$ & $29,22 \%$ & $20,63 \%$ & $13,60 \%$ & $8,53 \%$ & $5,16 \%$ & $3,04 \%$ \\
\hline & & $100,00 \%$ & $14,83 \%$ & $27,92 \%$ & $32,85 \%$ & $30,93 \%$ & $25,48 \%$ & $19,19 \%$ & $13,55 \%$ & $9,11 \%$ \\
\hline & & & $100,00 \%$ & $7,85 \%$ & $18,47 \%$ & $26.08 \%$ & $28,65 \%$ & $26,97 \%$ & $22,85 \%$ & $17,93 \%$ \\
\hline & & & & $100,00 \%$ & $4,15 \%$ & $11,73 \%$ & $19,33 \%$ & $24,26 \%$ & $25,69 \%$ & $24,19 \%$ \\
\hline & & & & & $100,00 \%$ & $2,20 \%$ & $7,24 \%$ & $13,64 \%$ & $19.26 \%$ & $22,67 \%$ \\
\hline & & & & & & $100,00 \%$ & $1,16 \%$ & $4,38 \%$ & $9,28 \%$ & $14,56 \%$ \\
\hline & & & & & & & $100,00 \%$ & $0.62 \%$ & $2,61 \%$ & $6,14 \%$ \\
\hline & & & & & & & & $100,00 \%$ & $0,33 \%$ & $1,53 \%$ \\
\hline & & & & & & & & & $100,00 \%$ & $0.17 \%$ \\
\hline obabilida & ciòn & & & & & & & & & $100,00 \%$ \\
\hline
\end{tabular}

Fuente: Elaboración propia

Tabla 11. Comparación de flujo de fondo libre en $t$ con el valor presente esperado $t+1$

\begin{tabular}{|c|c|c|c|c|c|c|c|c|c|c|}
\hline 0 & 1 & 2 & 3 & 4 & 5 & 6 & 7 & 8 & 9 & 10 \\
\hline 15448 & 26824 & 38784 & 51356 & 64573 & 78468 & 93075 & 108431 & 124574 & 141545 & 159386 \\
\hline . & 1 & 2 & 3 & 4 & 5 & 6 & 7 & 8 & 9 & 10 \\
\hline \multirow[t]{10}{*}{ - } & 118995 & 270864 & 493608 & 820301 & 1299454 & 2002218 & 3032946 & 4544693 & 6761939 & 10013924 \\
\hline & & - & 118995 & 270864 & 493608 & 820301 & 1299454 & 2002218 & 3032946 & 4544693 \\
\hline & & & - & - & 118995 & 270864 & 493608 & 820301 & 1299454 & 2002218 \\
\hline & & & - & - & - & - & 118995 & 270864 & 493608 & 820301 \\
\hline & & & & - & - & - & - & - & - & 270864 \\
\hline & & & & & - & - & - & - & - & - \\
\hline & & & & & & . & - & . & - & - \\
\hline & & & & & & & - & - & - & - \\
\hline & & & & & & & & - & - & - \\
\hline & & & & & & & & & - & - \\
\hline Compara & cada perio & lación (14) & & & & & & & & - \\
\hline
\end{tabular}

Fuente: Elaboración propia Moneda: \$AR

Tabla 12. Valor de la Opción Real para el componente forestal

\begin{tabular}{|c|c|c|c|c|c|c|c|c|c|c|}
\hline 0 & 1 & 2 & 3 & 4 & 5 & 6 & 7 & 8 & 9 & 10 \\
\hline \multirow[t]{11}{*}{ - } & 56014 & 60019 & 51486 & 40277 & 30034 & 21784 & 15533 & 10956 & 7674 & 5349 \\
\hline & - & - & 41867 & 59814 & 64137 & 60208 & 52379 & 43418 & 34830 & 27297 \\
\hline & & - & - & - & 34769 & 55883 & 67114 & 70002 & 67114 & 60848 \\
\hline & & & - & - & - & - & 30319 & 51979 & 66883 & 74745 \\
\hline & & & & - & - & - & - & - & - & 48563 \\
\hline & & & & & . & . & - & . & . & . \\
\hline & & & & & & . & . & . & . & . \\
\hline & & & & & & & - & . & - & - \\
\hline & & & & & & & & . & . & . \\
\hline & & & & & & & & & - & - \\
\hline & & & & & & & & & & - \\
\hline . & 53282 & 54308 & 80350 & 81947 & 100419 & 102140 & 116516 & 118215 & 112542 & 131497 \\
\hline
\end{tabular}

Fuente: Elaboración propia. Moneda: \$AR 
Tabla 13. Valor Opción Real del sistema entre el año 10 y 20, en gris se señala el turno

\begin{tabular}{|l|r|r|r|r|r|r|r|r|r|r|r|}
\hline Año & 10 & 11 & 12 & 13 & 14 & 15 & 16 & 17 & 18 & 19 & 20 \\
\hline VOR Forestal & - & & 447279 & 371156 & 301218 & 722951 & 643113 & 558124 & 983730 & 895519 & 1909532 \\
\hline VOR Ganadero & - & 44464 & 45889 & 68392 & 70531 & 86907 & 89240 & 84290 & 104609 & 100760 & 117660 \\
\hline VOR Total & - & 44464 & 493168 & 439548 & 371749 & 809858 & 732353 & 642414 & 1088339 & 996279 & 2027193 \\
\hline
\end{tabular}

Fuente: Elaboración propia. Moneda: \$AR

Además, el riesgo en el modelo vaN se incorporó íntegramente en el factor de actualización y los periodos fueron tratados sin flexibilidad alguna (solamente tala) y el resultado se encontró en parte influenciado por el impacto de la tasa ajustada por riesgo en el flujo de fondos esperado estático. En la figura 1 se expone el perfil de beneficios en ambos enfoques.

\section{DISCUSIÓN}

Se expuso un modelo alternativo al clásico método de descuento de flujos de fondos, para estimar la conveniencia económica-financiera y temporal de ejercer la opción de talar una masa forestal sujeta a un manejo silvopastoril, siendo esto correspondiente con la madurez del proyecto de inversión. Se propone aplicar la teoría de opciones reales para incorporar en el marco de decisión la posibilidad de diferir la tala y tomar en cuenta el proceso aleatorio del precio. La decisión consiste en comparar cada escenario proyectado versus el valor actual promedio de los escenarios del próximo ejercicio conforme a Plantinga (1998); en términos de opción financiera la decisión se asemeja a una barrier option del tipo knock-in.

En el caso analizado, el método de descuento de flujos de fondos sugiere talar en el año 11 obteniéndose un VAN de \$AR 511914 y utilizando una tasa ajustada por riesgo $\left(k\right.$ ) de $28,39 \%{ }^{12}$ siendo muy superior a las planteadas por Monicault y Delvalle (2009) y Fassola et al. (2004) los cuales no mencionan el criterio de selección y adicional por riesgo de mercado utilizado en el cálculo.

El resultado se debió a la alta sensibilidad negativa de los flujos al factor de descuento, reafirmando lo mencionado por Soares et al. (2005), además de que la variable precio se supuso constante en todo el periodo de proyección.

La aplicación del método de opciones reales, por el contrario, sugiere que el máximo valor se obtiene en el último periodo. Descartada esta opción, que no posee flexibilidad, el segundo momento óptimo se encontró en el año 18 con un ingreso con una VOR \$AR 1101 944, siendo superior al VAN en más de $100 \%$ ya que el mismo no permite el desarrollo futuro del bosque.

Estos valores coinciden con lo afirmado por Sant'Anna y Nogueira (2010) y Moreira et al. (2000). Además, se observó que la componente ganadera en el sistema no altera la decisión de tala ya que en forma independiente (sólo componente forestal) o como sistema se obtuvo el turno óptimo en el año 18.

\section{CONCLUSIONES}

Desde el punto de vista económico- financiero el enfoque de oR presenta importantes ventajas en contraposición al clásico VAN debido a su robustez, ya que incorpora flexibilidad estratégica y comporta- 
miento estocástico de los precios con base en series históricas; de esta manera elimina el uso de la tasa ajustada por riesgo.

Esta cuestión no se tiene en cuenta en el VAN, siendo la tasa ajustada por riesgo constante en la vida del proyecto, por lo cual supone un escenario constante en todo el horizonte de planificación, siendo esto un enfoque muy limitado, en especial tratándose de proyectos forestales, dado que presentan horizontes temporales extensos.

\section{REFERENCIAS}

Bettinger, P., K. Boston, J.P. Siry y D.L. Grebner. 2009. Forest management and planning. 1a. ed. Academic Press, California. 360 p.

Black, F. y M. Scholes. 1973. The pricing of options and corporate liabilities. Journal of Political Economy 81(3):637-659.

Blasco, C., S. Carenzo y E. Astrada. 2005. Evaluación de un sistema silvopastoril sobre vinales en Formosa, Argentina. Revista Iberoamericana de Economía Ecológica 2(1):57-68.

Brennan, M. y E. Schwartz. 1985. A new approach to evaluating natural resource investment. Midland Corporate Financial Journal 1(3):37-47.

Cerruto R., C. Leite, J. Gonçalves y M. Lopez da Silva. 2007. Estimativa do abatimento de erosão aportado por um sistema agrossilvipastoril e sua contrubução econômica. Revista Árvore 31(2):285-293.

Chidiak, M., A. Moreyra y C. Greco. 2003. Captura de carbono y desarrollo forestal sustentable en la Patagonia
Argentina: Sinergias y Desafíos. Documento CENIT-CEPAL-UDESA. $136 \mathrm{p}$.

URL:http://www.territorioscentroamericanos.org/ecoagricultura/Documents/ capturadecarbonoydesarrolloforestal.pdf (último acceso 08/08/2013).

Colcombet, L., E. Crechi, A. Keller, N. Pachas, H. Fassola, S. Lacorte y J. Esquivel. 2010. Comparación preliminar de resultados financieros y económicos de proyectos forestales, ganaderos y silvopastoriles en Misiones. Acta $14^{\text {as }}$ Jornadas Técnicas Forestales y Ambientales. UNAM - EEA Montecarlo, INTA. 86 p.

Copeland, T., T. Koller y J. Murrin. 2000. Valuation: measuring and managing the value of companies. $3^{\mathrm{a}} \mathrm{ed}$. Wiley. Nueva York. 490 p.

Cox, J., S. Ross y M. Rubinstein. 1979. Option pricing: a simplified approach. Journal of Financial Economics 7(3):229-263.

Crechi, E., R. Fernández, A. Friedl y J. Falher. 2005. Efectos de raleo en Pinus taeda, Norte Misionero. Revista Idia 11(8):41-45.

Crechi, E., H. Fassola y A. Friedl. 2005. Simulador Forestal. Revista Idia 11(8):46-50.

Damodaran, A. 2002. Investment Valuation. $2^{\mathrm{a}}$ ed. Wiley. Nueva York. 992 p.

Dixit, A. y R. Pindyck. 1994. Investment under uncertainty. $1^{\text {a }}$ ed. Pricenton University Press. Nueva Jersey. $476 \mathrm{p}$.

Fassola, H., S. Lacorte, J. Esquivel, F. Colcombet, F. Moscovich, E. Crechi, N. Pachas y A. Keller. 2004. Sistemas silvopastoriles en Misiones y NE 
de Corrientes y su entorno de negocio. Directorio de información Instituto Nacional del Tecnología Agropecuaria (INTA).

URL:http://anterior.inta.gob.ar/montecarlo/info/documentos/forestales/ sistsilvopastoril.PDF (último acceso 08/08/2013).

Grainger, M. 1968. Problems affecting the use of Faustmann's formula as a valuation tool. New Zealand Journal of Forestry (1968) 13(2):168-183.

Hull, J. 2005. Futures, options and other derivatives. $6^{a}$ ed. Prentice Hall. Nueva Jersey. 816 p.

Kulatilaka, N. 1988. Valuing the flexibility of flexible manufacturing systems. IEEE Transactions in Engineering Management 35(4):250-257.

Malliaris, A. G. y W.A. Brock. 1982. Stochastic methods in economics and finance. $1^{\mathrm{a}}$ ed. Holanda del Norte, Amsterdam. 317 p.

Margrabe, W. 1978. The value of an option to exchange one asset for another. Journal of Finance 33(1):177-186.

Markowitz, H. 1991. Portfolio selection: efficient diversification of investments. 2a ed. Blackwell Publishers. Cambridge, Massacchusetts. 384 p.

Mason, S. y R. Merton. 1985. The Role of contingent claims analysis in corporate finance. Division of Research, Graduate School of Business Administration, Harvard University. Home-wood Irwin, Nueva York. 65 p.

Mc Donald, R. y D. Siegel. 1981. Option pricing when the underlying asset is non-stored. Discussion Papers number 512. Center for Mathematical Studies in Economics and Management Science, Northwestern University. Evaston, Illinois. 12 p.

Merton, R. 1973. The theory of rational options princing. The Bell Journal of Economics and Management Science 4(1):141-183.

Miller, R. A. y K. Voltaire. 1983. A stochastic analysis of the tree paradigm. Journal of Economic Dynamics \& Control 6(1):371-386.

Milanesi, G. y H. Vigier. 2010. Árboles de decisión, opciones reales y enfoque integrado en mercados completos e incompletos. Anales Asociación Argentina de Economía Política. Buenos Aires. p:1-30.

Milanesi, G. 2011. Opciones reales: teoría y casos prácticos. $1^{\text {a }}$ ed. Induvio, Buenos Aires. 156 p.

Milanesi, G., G. Woitschach y D. Broz. (2012). Aplicación de la teoría de opciones reales a la determinación del momento óptimo de cosecha forestal. Rev. FCA UNCUYO. 44(2):6578.

Monicault, L. A. y P. Delvalle. 2009. Evaluación Económico-Financiero de un Sistema Silvopastoril (SSP). Documeto técnico INTA online. URL:http:// inta.gob.ar/documentos/evaluacioneconomico2013financiero-de-un-sistema-silvopastoril-ssp/ (último acceso 08/08/2013).

Moreira A., E. Reis, K. Rocha y L. CarvaIho. 2000. A valoração das concessões nas florestas nacionais da Amazônia: uma abordagem com 
opções reais. Pesquisa e Planeamento Econômico 30(3):327-354.

Mun, J. 2005. Real options analysis: tools and techniques for valuing strategic investment and decisions. $2^{\mathrm{a}} \mathrm{ed}$. Wiley. Nueva York. 704 p.

Musálem, M.A. 2002. Sistemas agrosivopastoriles: una alternativa de desarrollo rural sustemtable para el trópico mexicano. Revista Chapingo 8(2):91100.

Myers, S. y S. Majd. 1990. Abandonment value and project life. Advances in Futures and Options Research 4:1-21.

Myers, S. 1977. Determinants of corporate borrowing. Journal of Financial Economics 5(2):147-175.

Paddock, J., D. Siegel y J. Smith. 1988. Option valuation of claims on physical assets: the case of Offshore Petroleum Lease. Quaterly Journal of Economics 103(3):479-508.

Pindyck, R. 1988. Irreversible investment, capacity choice and the value of the firm. American Economic Review 78(5):969-985.

Plantinga, A. 1998. The optimal timber rotation: an option value approach. Forest Science, Society of American Foresters 44(2):192-202.

Pratt, S. y R. Grabowski. 2008. Cost of capital: applications and examples. $3^{a}$ ed. John Wiley \& Sons Nueva Jersey. $778 \mathrm{p}$.

Prodan, M., R. Peters, F. Cox y P. Real. 1997. Mensura Forestal. $1^{\mathrm{a}}$ ed. Costa Rica. Instituto Interamericano para la Agricultura - Gesellschaft für Zusammenarbeit (IICA-GTZ). $511 \mathrm{p}$.
Rocabert, J. (2007). Los criterios valor actual neto y tasa interna de rendimiento. Revista electrónica sobre la enseñanza de la Economía Pública 2(1):1-11.

Sant'Anna, A. y J. Nogueira. 2010. Valoração econômica dos serviços abientais de florestas nacionais. Revista de Administração e Negócios da Amazônia 2(1):82-108.

Schneider, P. 2002. Manejo florestal: planejamento da produção florestal. Santa María, Universidade Federal de Santa Maria, Centro de Ciências Rurais, Departamento de Ciências Florestais. $492 \mathrm{p}$.

Smit, H. 1997. Investment analysis of offshore concessions in The Netherlands. Financial Management 26(2):5-17.

Smith, J. 2005. Alternative approach for solving real options problems. Decision Analysis 2(2):89-102.

Soares, T.S., R. M. Carvalho, A. Bartolomeu do Vale. 2003. Avalição econômica de um povoamento de Eucalyptus grandis destinado a multiprodutos. Revista Árvore. 27(5): 689-694.

Thomson, T.A. 1992. Optimal forest rotation when stumpage prices follow a difusion process. Land Economics 68(3):329-342.

Trigeorgis, L. y S. Mason. 1987. Valuing Managerial Flexibiliy. Midland Corporate Finance Journal 5(1):14-21.

Trigeorgis, L. 1988. A conceptual options framework for capital budgeting. Advances in Futures and Options Research 4(3):145-167. 
Trigeorgis, L. 1997. Real options: managerial flexibility and strategy in resource allocations. $2^{\mathrm{a}}$ ed. MIT Press, Cambridge. 427 p.
Yin, R. y D. Newman. 1997. When to cut a stand of trees? Natural Resource Modeling 10(3):251-261. vopastoriles: análisis de inversión. Madera y Bosques 19(3):81-98. 